This item was submitted to Loughborough's Research Repository by the author.

Items in Figshare are protected by copyright, with all rights reserved, unless otherwise indicated.

\title{
A high-resolution domestic building occupancy model for energy demand
} simulations

PLEASE CITE THE PUBLISHED VERSION

PUBLISHER

(C) Elsevier

VERSION

AM (Accepted Manuscript)

LICENCE

CC BY-NC-ND 4.0

\section{REPOSITORY RECORD}

Richardson, lan, Murray Thomson, and David Infield. 2019. "A High-resolution Domestic Building Occupancy Model for Energy Demand Simulations”. figshare. https://hdl.handle.net/2134/4757. 
This item was submitted to Loughborough's Institutional Repository (https://dspace.lboro.ac.uk/) by the author and is made available under the following Creative Commons Licence conditions.

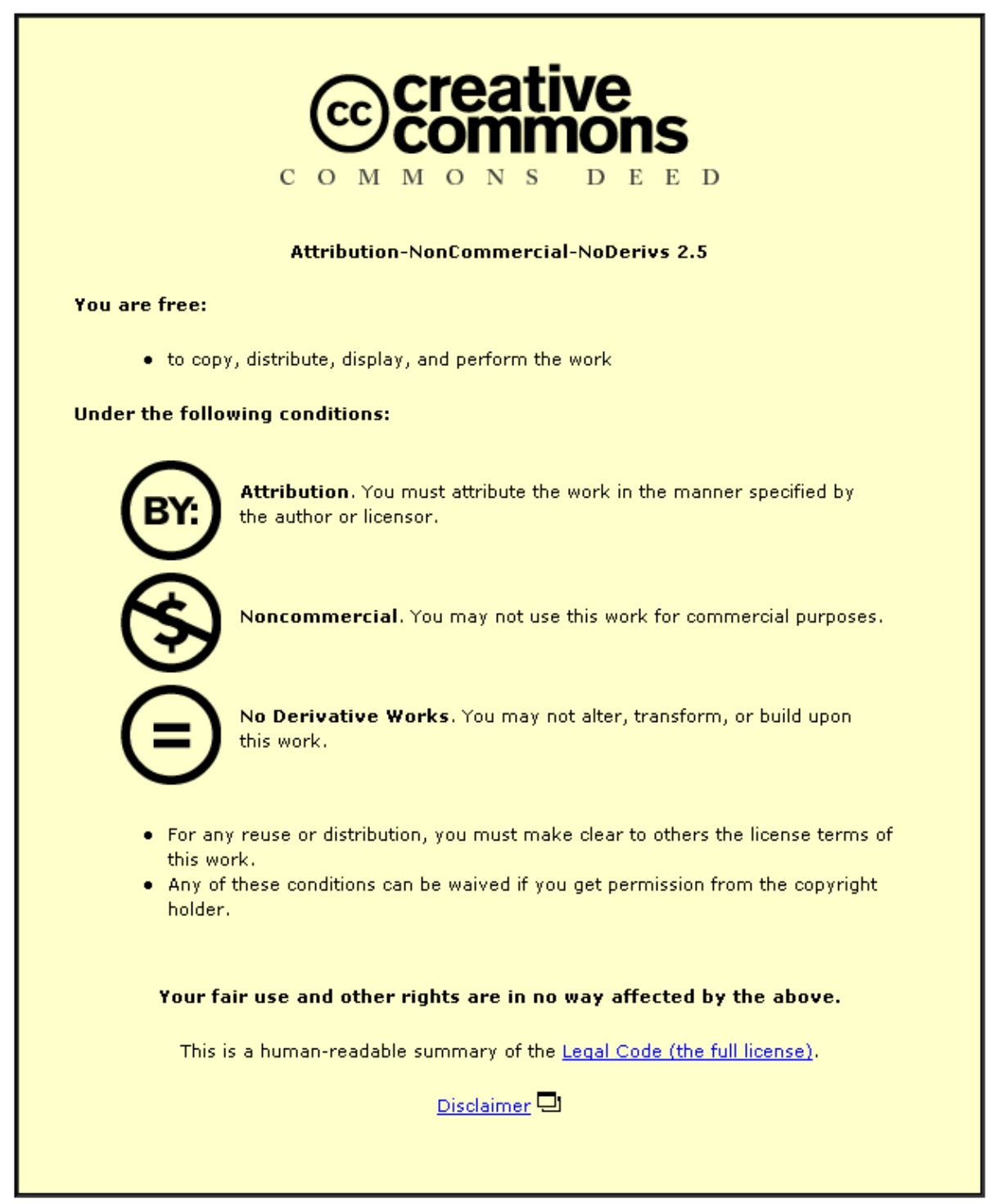

For the full text of this licence, please go to: http://creativecommons.org/licenses/by-nc-nd/2.5/ 


\title{
A high-resolution domestic building occupancy model for energy demand simulations
}

\author{
Ian Richardson ${ }^{1^{*}}$, Murray Thomson $^{1}$ and David Infield ${ }^{2}$ \\ ${ }^{1}$ CREST (Centre for Renewable Energy Systems Technology), \\ Department of Electronic and Electrical Engineering \\ Loughborough University, Leicestershire LE11 3TU, UK \\ ${ }^{2}$ Department of Electronic \& Electrical Engineering, University of Strathclyde, UK \\ *Corresponding author: Tel. +44 1509 635326. Email address: I.W.Richardson2@lboro.ac.uk
}

\begin{abstract}
Energy use in the home is a major source of carbon emissions and is highly dependent on the activities of the residents. More specifically, the timing of energy use, particularly electricity, is highly dependent on the timing of the occupants' activities. Thus, in order to model domestic demand profiles with high temporal resolution, for example in the context of designing and assessing demand side management systems (including the time-shifting of demand), it is of great benefit to take account of residents' behaviour in terms of when they are likely to be using household appliances, lighting and heating. This paper presents a thorough and detailed method for generating realistic occupancy data for UK households, based upon surveyed timeuse data describing what people do and when. The approach presented generates statistical occupancy time-series data at a ten-minute resolution and takes account of differences between weekdays and weekends. The model also indicates the number of occupants that are active within a house at a given time, which is important for example in order to model the sharing of energy use (shared use of appliances etc.) The data from the model can be used as input to any domestic energy model that uses occupancy time-series as a base variable, or any other application that requires detailed occupancy data. The model has been implemented in Excel and is available for free download.
\end{abstract}

Keywords: Domestic occupancy; energy modelling 


\section{Introduction}

Detailed electricity and thermal demand profiles for domestic housing are an important prerequisite for the accurate analysis of new low-carbon technologies and strategies, such as distributed generation and demand side management.

The use of appliances, lighting, heating and domestic hot water within a building varies considerably with respect to time, mainly in accordance with the activity of the building residents. In the domestic sector, it depends not only on the number of people who live at a property but also on whether they are at home and active (i.e. not asleep). Several existing energy-use models support this premise, one example being the Tyndall Centre report "Microgrids: distributed on-site generation" [1], which comments that "electricity load profile depends mainly on the household size and occupancy pattern."

We define an "active occupant" as a person who is in the house and not asleep.

For accurate demand modelling, it is also important to account for the sharing of appliances lighting and heating. For example, doubling the number of active occupants in a house is unlikely to double the lighting demand, since occupants will share lighting in common rooms. Sharing is dependent on the number of active occupants in a house at a given time and also on the appliance type in question.

Unfortunately, and perhaps unsurprisingly, large data sets describing the number of active occupants in houses are not readily available. Paatero and Lund [2] discuss the requirement for detailed data about households when constructing "bottom-up" electrical energy-use models. Such models aggregate individual appliance loads, over a time-series and benefit from "grassroot level consumption details", or detail on "statistical averages" in order to configure the model. Other work to which Paatero and Lund refer is Capasso et al. [3], which details a complex residential load model that requires the accounting of occupant data such as the "availability at home profile" for each occupant. Stokes et al. [4] comment that "Taking account 
of these [occupancy] patterns would improve the modelling of diversity." Similarly, Yao and Steemers [5] say that "the load profile depends very much on the occupancy pattern." Thus, it is clear that the ability to generate synthetic occupancy data is of considerable benefit in the context of energy-use modelling; it may also be of value in other areas of research.

This paper presents a model that generates synthetic active occupancy data, based upon survey data of people's time-use in the United Kingdom [6]. A Markov-Chain technique is used to generate the synthetic data such that it has the same overall statistics as the original survey data. 


\section{Time Use Survey (TUS) Data}

A large survey of how people use their time was conducted in the United Kingdom in year 2000

[6] and is known as the Time Use Survey (TUS). It contains detailed 24-hour diaries, completed at ten-minute resolution by many thousands of participants. The data includes the location of the participants, at each ten-minute diary period, and can thus be used to identify the number of active occupants in a house. (A value of ' 2 ' in the location field in the data indicates that the participant is at home and is not asleep, away at study or at work.) 


\subsection{Individual Occupancy Profiles}

Fifty example active occupancy profiles, taken from the TUS data, are shown in Fig. 1. Each horizontal bar indicates that the person is active in the property at that time. As expected, there is minimal activity during the night (00:00 - 07:00), some activity (at home) during the day and most activity during the evening.

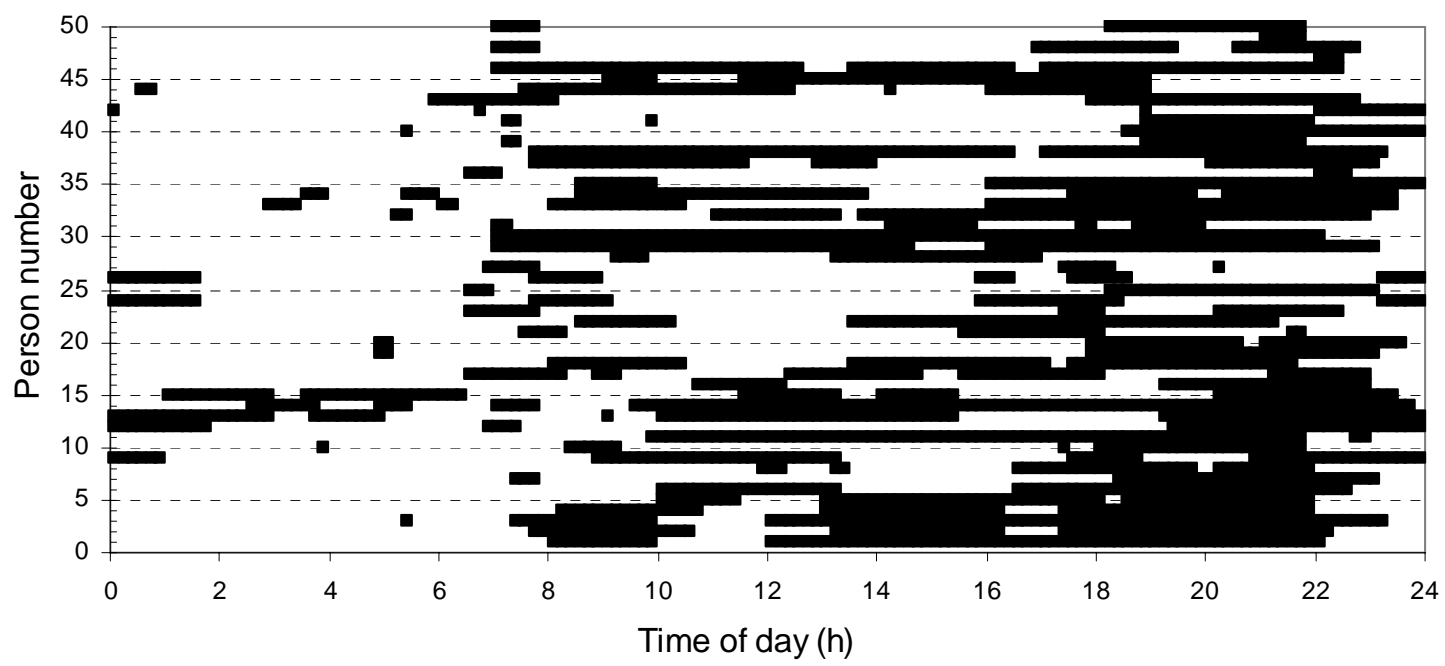

Fig. 1. Fifty example active occupancy profiles drawn from the TUS data 


\subsection{House Occupancy Profiles}

It is natural that the activity of people living at the same address will be correlated and it is important that this characteristic is included in the occupancy model so that sharing of appliances etc can be properly represented in subsequent energy-use modelling. Fortunately, the TUS data indicates which people live at the same address and thus provides a direct means of knowing the number of active occupants in each house at each ten-minute interval.

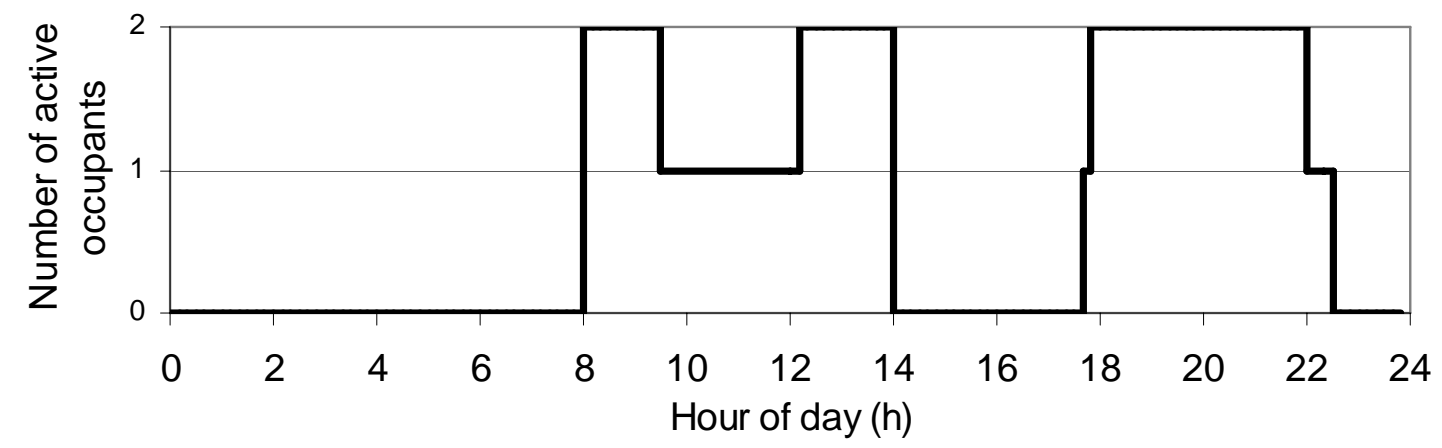

Fig. 2. An example active occupancy pattern for $15^{\text {th }}$ October 2000

An example house from the TUS data is presented in Fig. 2. It can be seen that both occupants become active at 08:00 (probably they get up) and that they both leave the house (or less likely go to sleep) at 14:00. This correlated behaviour is a common feature within the survey data. 


\subsection{Aggregate Occupancy Profiles}

The proportion of houses, with at least one active occupant for each ten-minute diary period for the whole TUS data set, was summed into weekday and weekend groups and the results are shown in Fig. 3. The curves display several expected features including: very low levels of activity throughout the night, delayed getting up at weekends, and peaks corresponding to breakfast, lunch and evening. Moreover, it is interesting to note that this occupancy profile has a striking similarity to a typical domestic electricity demand profile [7], which clearly adds considerable weight to the earlier assertion that electricity demand should be based on active occupancy.

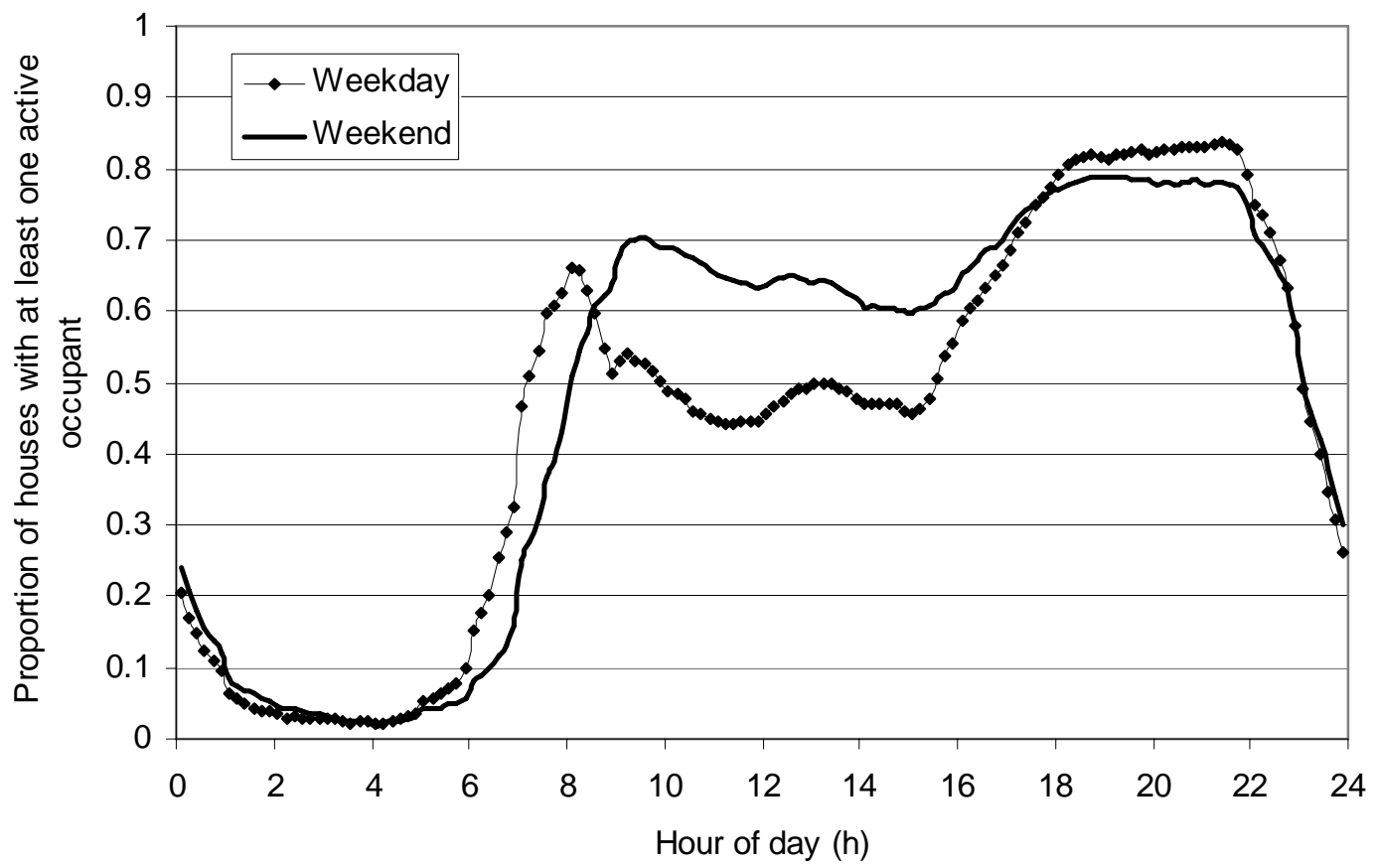

Fig. 3. Aggregated active occupancy for all survey participants by weekday and weekend days 


\section{Modelling}

The aim of the model is to generate stochastic occupancy data with the same characteristics as the Time Use Survey data, thus allowing much larger data sets to be created. The first-order Markov-Chain technique, a well-established stochastic method of data generation $[8,9,10]$, was selected for the purpose.

The TUS data is for ten-minute periods, and thus the active occupancy time-series data for one day for a given house (such as that shown in Fig. 2) comprises 144 integer numbers, which are known as "states" and where the integer is the number of active occupants for the ten minute period in question. The concept of the first-order Markov-Chain technique is that each state is dependent only on the previous state together with the probabilities of the state changing. These probabilities are held in "transition probability matrices", an example of which is presented in Table 1.

The example is for a one-person household, starting at midnight. If the person is asleep at 00:00, then they will very likely still be asleep at 00:10. This in represented by the 0.994 probability in the top-left cell. Alternatively, if the person is awake at 00:00, then the probability that they will retire by $00: 10$ is 0.207 , as shown in the bottom-left cell. (For simplicity, the possibility of the person entering or leaving the house is omitted from the above discussion.) Ten minutes later, the concept is similar but the probabilities are slightly altered. Thus, 144 transition probability matrices are required in order to represent a whole day. Note that in the very simplest Markov models, the transition matrix is unchanging. However, such models would not capture the evident time dependence of the process.

In order to generate the synthetic data, a random number (uniform from 0 to 1 ) is picked at each time step and used, together with the appropriate transition probability matrix, and with the state at the current time step, to determine the state at the next time step. This is commonly known as the Markov-Chain Monte-Carlo technique $[9,10]$. A two-person household is represented 
similarly but with 3-by-3 transition probability matrices. Likewise, larger households require larger matrices. 
3.1 Building the Transition Probability Matrices

The transition probability matrices are derived from the TUS data as illustrated by the example presented in Table 2. There are 144 such calculations to complete the representation of a whole day.

For the purpose of the occupancy model described in this paper, the TUS source data was categorised by:

- the number of household residents (1 to 6 );

- whether the day was a weekday or a weekend day.

For some households in the TUS data, the number of available diaries is less than the recorded number of household residents (for example due to young children). In these cases, the model is based on available diaries only.

The 144 calculations exemplified by Table 2 were repeated for each category, yielding a set of matrices, as summarised in Table 3 . 


\subsection{Start States}

In order to generate the Markov chain, it is necessary to provide a start state: how many active occupants are in the house at 00:00. This is of course random but should match the probabilities found in the original TUS data. Fig. 4 shows the probability distributions derived from the TUS data. As expected, the majority of occupants are inactive at 00:00.

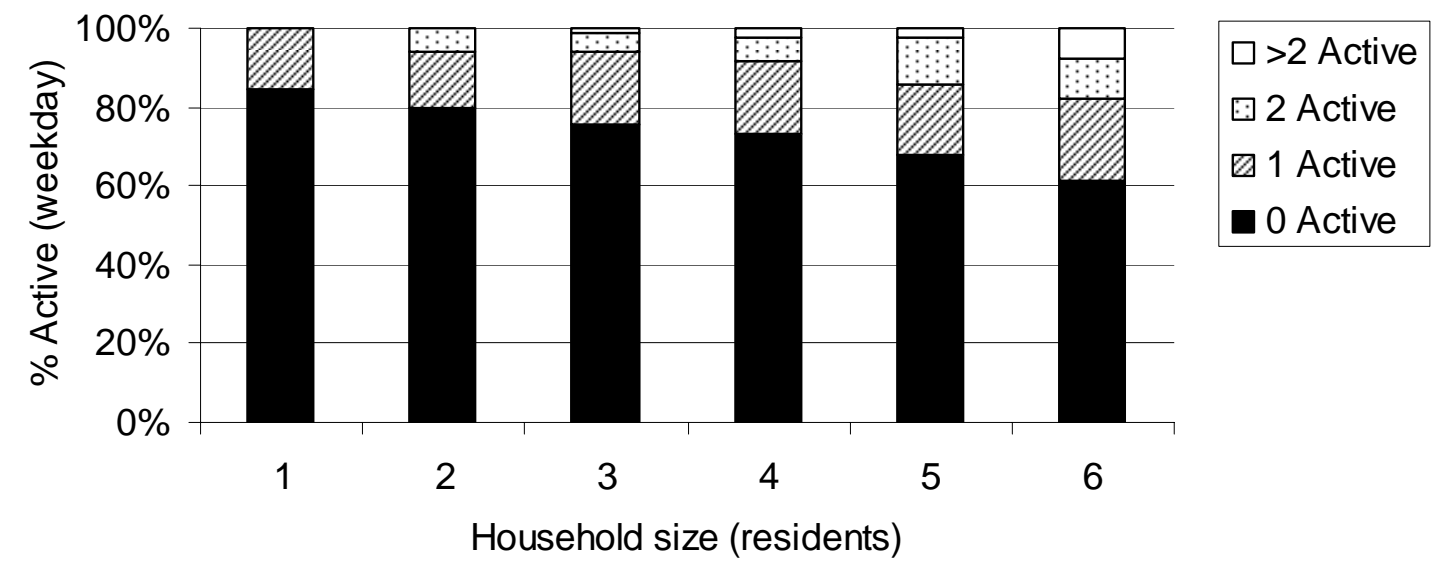

Fig. 4. Percentage distribution of active occupancy states at 00:00

\subsection{Generating the Data}

The start state is chosen by picking a random number of active occupants from a probability distribution as shown in Fig 4. Subsequent states in the chain are determined by picking a random number for each time step and using this with the appropriate transition probability matrix (given the day of the week and the total number of residents in the house).

\subsection{Downloadable Model}

An example implementation of the model, in the form of a Microsoft Excel workbook, has been made available for free download [11]. It contains the transition probability matrices, the start state distributions and a Visual Basic implementation of the algorithm. The source code is open and may be readily adapted for specific application, with due acknowledgment to the authors. 


\section{Verification and Use of the Model}

4.1 Individual House Occupancy Simulations

The output from four example runs of the model, for a two-person house on a weekday, is shown in Fig. 5. Each run is different because of the random numbers used in the stochastic generation but all runs are based on the same transition probability matrices and thus exhibit similar characteristics. It can be seen that occupants typically get up in the morning, leaving the house shortly afterwards, possibly returning for lunch and then returning for the evening. 

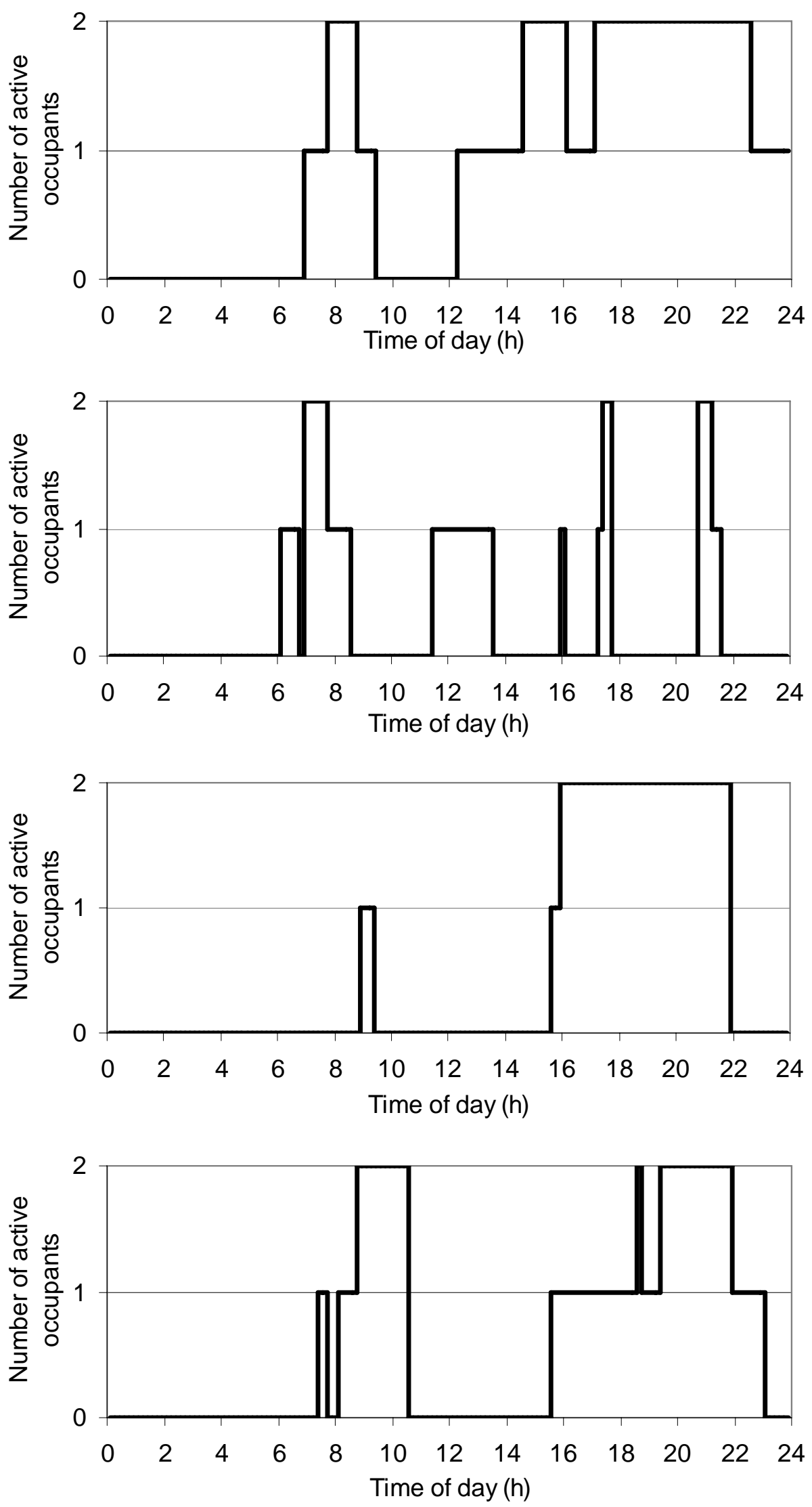

Fig. 5. Four occupancy model example run results (2 resident household, weekday) 
Comparison of the occupancy patterns shown in Fig. 5 to that shown in Fig. 2 give a first indication that the model is working as expected: showing low levels of activity at night and increased levels in the mornings and evenings. In order to validate the model more fully, it was run a very large number of times and the statistics of its output compared against the original TUS data. 
A comparison of the aggregated model output against TUS data is shown in Fig. 6 for two resident households, for both weekend and weekday scenarios. This shows the proportion of active occupants in 2000 houses from the TUS data, compared to the proportion of active occupants in 10000 houses as generated by the model.

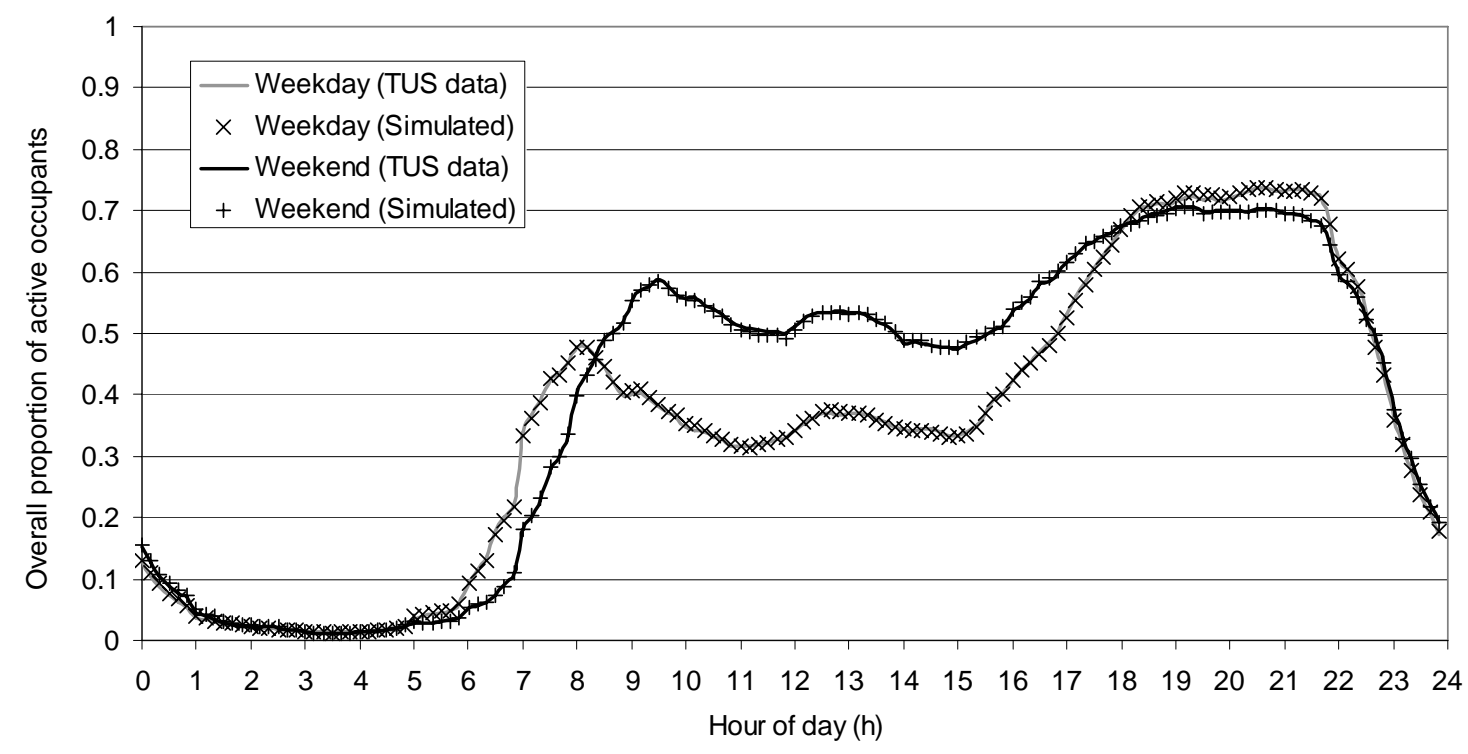

Fig. 6. Comparison of simulated and surveyed data for a weekday (2 resident household)

In terms of the aggregated active occupancy profile for a large number of houses, Fig. 6 confirms that the simulation output is generating data with an almost identical active occupancy profile to the TUS data. The close correlation between the model output and the TUS data is also seen when performing comparisons for houses with other numbers of residents. 
4.3 Verification of Transitions

A further verification of the model output is to compare the number of occupancy transitions for each ten minute period of the day, as generated by the model, against the TUS data. The number of people becoming active and inactive is shown in Fig 7, for a sample size of 1000 two-resident households.
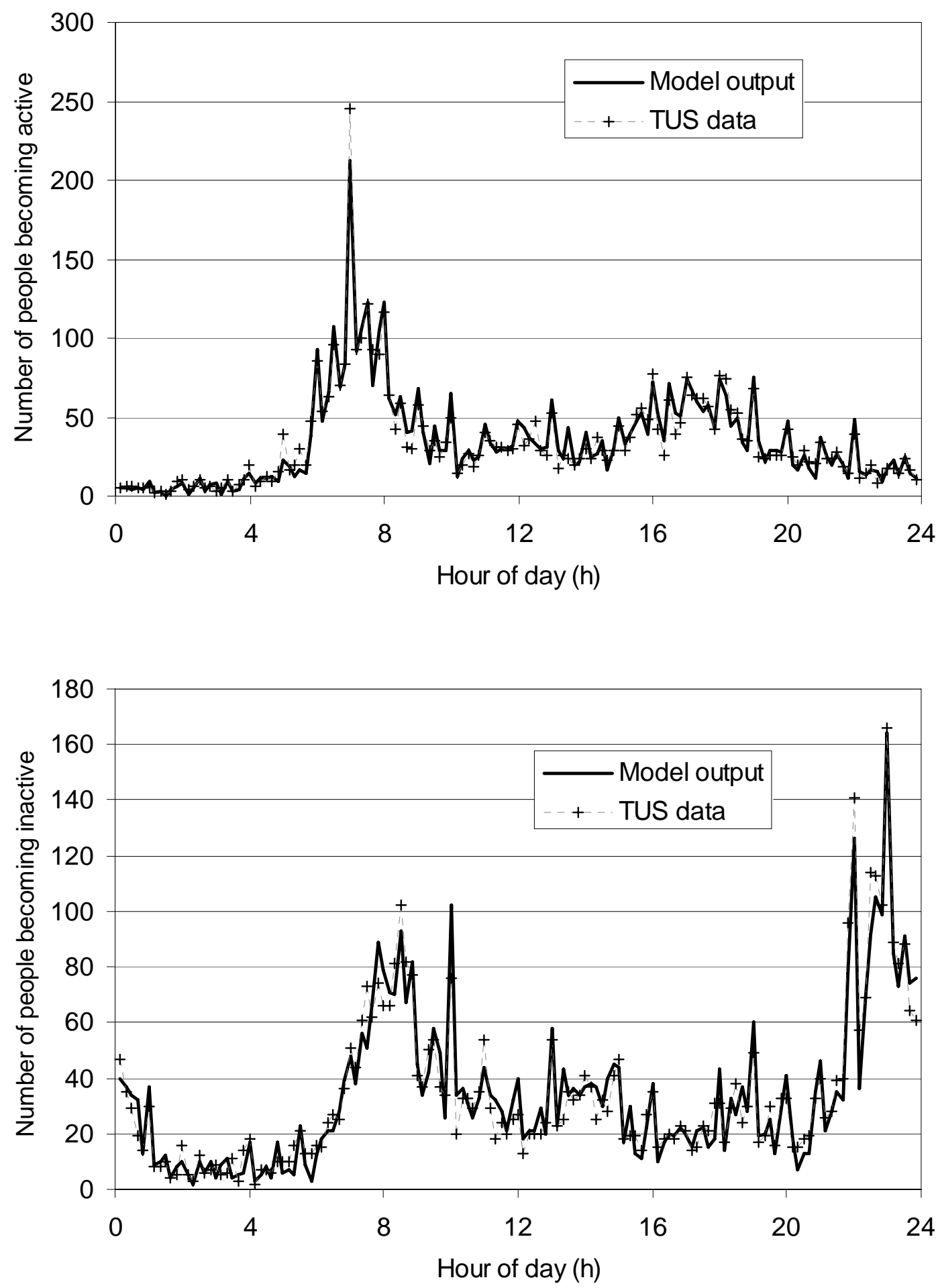

Fig. 7. Number of people becoming active and inactive (2 resident household, weekday) 
Transitions to an active occupancy state, shown in Fig. 7, occur with two peaks: the first at breakfast time and the second in the late afternoon. Transitions to an inactive state occur also with two peaks: one at breakfast time and the second at the end of the evening. Moreover, Fig. 7 shows that model generates data that has very similar transition characteristics to the TUS data, especially given that the number of people becoming active and inactive, at each time step, is a subset of the total of 2000 residents in this example. 
A verification of the model's simulation of correlated occupancy changes is shown in Fig. 8.

This plot shows the number of correlated occupancy changes for 1000 two-resident households and represents the number of times that either both residents become active or inactive at the same time.

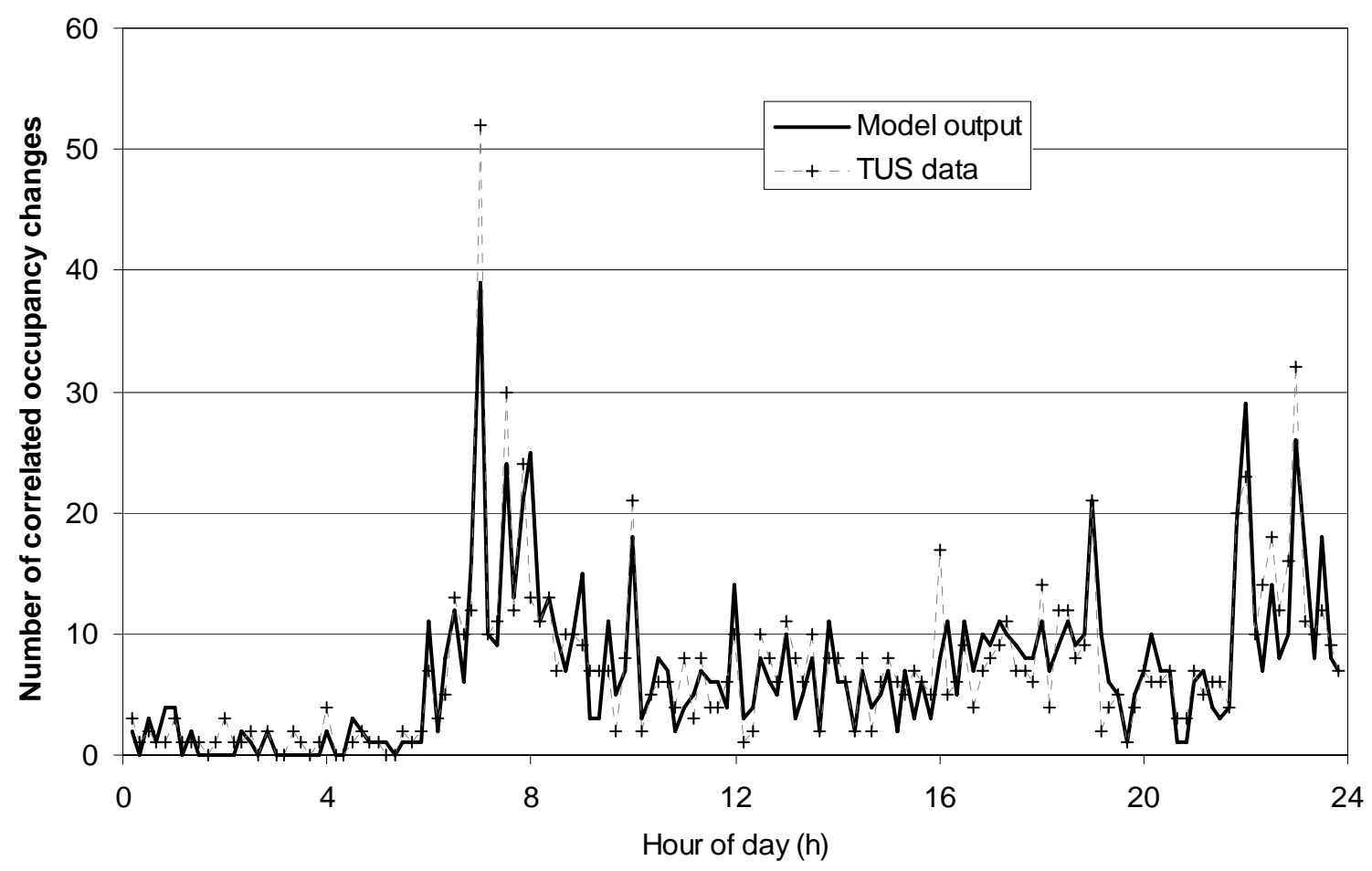

Fig. 8. Number of correlated occupancy changes (2 resident household, weekday)

The peak in the early morning shows that people tend to get up and leave the house together and this can be seen in both the TUS and model output data. Again, a very good correlation between the model and the original data is apparent. 


\subsection{Discussion}

The preceding comparisons indicate that the model performs very well in terms of producing data with statistical characteristics similar to the original TUS data. It is also very computationally efficient: large quantities of data may be generated very quickly. One reason for this is that to generate the next state in the chain, it is only necessary to generate one random number and perform a lookup and comparison against the appropriate transition probability matrix.

The TUS survey data provides diaries for individual days only and not a number of consecutive days. Although the model could be used to generate data spanning multiple days, it will not take into account patterns of consistency from day to day. For example, in the real world, a person who sets off to work at 08:00 is likely to do similar the next day, whereas multiple runs of the model will not reflect this. It would perhaps be possible to infer such patterns from the data, but this would be largely speculative and is not considered a satisfactory enhancement. 


\section{Conclusions}

A model has been presented that provides a stochastic simulation of active occupancy patterns in UK households. This model is based upon the UK 2000 Time Use Survey (TUS) data set and uses a Markov-Chain technique to generate further data with statistical characteristics that match the original.

The technique of building transaction probability matrices from the source data and using these to generate synthetic data series is very effective and computationally efficient. The statistical characteristics of the original TUS data and simulation output correlate very closely.

An implementation of the model, in the form of a Microsoft Excel workbook, is available for free download and may be adopted and adapted for specific application.

The occupancy patterns generated by this model are intended to be used in applications such as high-resolution domestic energy-use models, where diversity and sharing are important factors. With this in mind, the TUS source data was categorised by: weekday/weekend and by the number of residents in the household. For other applications or for more detailed energyuse modelling, alternative categorisation could be implemented. For example, socio-economic classification, which is also available in the TUS data, would enable the model to be tailored to particular regional housing characteristics or to other specific requirements. 


\section{Acknowledgement}

This work was supported by E.ON UK and by the Engineering and Physical Sciences Research Council, UK, within the Supergen 3 Highly Distributed Power Systems Consortium, grant number GR/T28836/01. 


\section{References}

[1] S. Abu-Sharkh, R. Li, T. Markvart, N. Ross, P. Wilson, R. Yao, K. Steemers, J. Kohler, R. Arnold, Microgrids: distributed on-site generation, Technical Report 22, Tyndall Centre for Climate Change Research, 2005

[2] J. V. Paatero, P.D. Lund, A model for generating household electricity load profiles, International Journal of Energy Research 30 (5) (2006) 273-290

[3] A. Capasso, W. Grattieri, R. Lamedica, A. Prudenzi, A bottom-up approach to residential load modelling, IEEE Transactions on Power Systems 9 (2) (1994) 957-964

[4] M. Stokes, M. Rylatt, K. Lomas K, A simple model of domestic lighting demand, Energy and Buildings 36 (2) (2004) 103-116

[5] R. Yao, K. Steemers, A method of formulating energy load profile for domestic buildings in the UK, Energy and Buildings 37 (6) (2005) 663-671

[6] Ipsos-RSL and Office for National Statistics, United Kingdom Time Use Survey, 2000 [computer file]. 3rd Edition. Colchester, Essex: UK Data Archive [distributor], September 2003. SN: 4504

[7] Elexon, Load Profiles and Their Use in Electricity Settlement, http://www.elexon.co.uk/participating/MarketGuidance/IndustryHelpDesks/ElexonHelpDesk/faqs .aspx\#WhatisaLoadProfile, consulted 23 $3^{\text {rd }}$ November 2007.

[8] E.W. Weisstein, Markov Chain, From MathWorld--A Wolfram Web Resource. http://mathworld.wolfram.com/MarkovChain.html, consulted 23 ${ }^{\text {rd }}$ November 2007. 
[9] W.R. Gilks, S. Richardson, D. Spiegelhalter, Markov Chain Monte Carlo in Practice, Chapman and Hall, 1995

[10] D. Gamerman, Markov Chain Monte Carlo - Stochastic Simulation for Bayesian Inference, Chapman and Hall, 1997

[11] I. Richardson, M. Thomson, Domestic active occupancy model - simulation example, Loughborough University Institutional Repository, http://hdl.handle.net/2134/3112, 2008 


\section{Figure Legends}

Fig. 1. Fifty example active occupancy profiles drawn from the TUS data

Fig. 2. An example active occupancy pattern for 15th October 2000

Fig. 3. Aggregated active occupancy for all survey participants by weekday and weekend days

Fig. 4. Percentage distribution of active occupancy states at 00:00

Fig. 5. Four occupancy model example run results (2 resident household, weekday)

Fig. 6. Comparison of simulated and surveyed data for a weekday (2 resident household)

Fig. 7. Number of people becoming active and inactive (2 resident household, weekday)

Fig. 8. Number of correlated occupancy changes (2 resident household, weekday) 


\section{Tables}

Table 1

Example transition probability matrix for a one-person household

\begin{tabular}{|c|c|c|c|}
\cline { 2 - 4 } \multicolumn{1}{c|}{} & \multicolumn{2}{c|}{$\begin{array}{c}\text { Next State } \\
\text { (at 00:10) }\end{array}$} \\
\cline { 2 - 4 } \multicolumn{1}{c|}{} & Active Occupants & $\mathbf{0}$ & 1 \\
\hline \multirow{3}{*}{$\begin{array}{c}\text { Current State } \\
\text { (at 00:00) }\end{array}$} & 0 & 0.994 & 0.006 \\
\cline { 2 - 4 } & 1 & 0.207 & 0.793 \\
\hline
\end{tabular}


Table 2

Example calculation of the Transition Probability Matrix at 00:00 - 00:10 for one-person households on weekdays

\begin{tabular}{|c|c|c|c|c|}
\hline \multicolumn{2}{|c|}{$\begin{array}{c}\text { Number of active } \\
\text { occupants }\end{array}$} & \multirow{2}{*}{\multicolumn{2}{|c|}{$\begin{array}{l}\text { Number of occurrences in the TUS } \\
\text { data }\end{array}$}} & \multirow{3}{*}{$\begin{array}{l}\text { Transition Probability } \\
1428 / 1436=0.994\end{array}$} \\
\hline At $00: 00$ & At $00: 10$ & & & \\
\hline 0 & 0 & 1428 & \multirow{2}{*}{$1428+8=1436$} & \\
\hline 0 & 1 & 8 & & $8 / 1436=0.006$ \\
\hline 1 & 0 & 55 & \multirow{2}{*}{$55+211=266$} & $55 / 266=0.207$ \\
\hline 1 & 1 & 211 & & $211 / 266=0.793$ \\
\hline
\end{tabular}


Table 3

Transition Probability Matrix Sizes for the Occupancy Model

\begin{tabular}{|c|c|c|c|}
\hline & $\begin{array}{l}\text { Household size } \\
\text { (residents) }\end{array}$ & $\begin{array}{c}\text { Transition Probability Matrix for } \\
\text { each time step }\end{array}$ & $\begin{array}{c}\text { Overall Transition } \\
\text { Probability Matrices }\end{array}$ \\
\hline \multirow{6}{*}{ Weekday } & 1 & $2 \times 2$ & $2 \times 2 \times 144$ \\
\hline & 2 & $3 \times 3$ & $3 \times 3 \times 144$ \\
\hline & 3 & $4 \times 4$ & $4 \times 4 \times 144$ \\
\hline & 4 & $5 \times 5$ & $5 \times 5 \times 144$ \\
\hline & 5 & $6 \times 6$ & $6 \times 6 \times 144$ \\
\hline & 6 & $7 \times 7$ & $7 \times 7 \times 144$ \\
\hline \multirow{6}{*}{ Weekend } & 1 & $2 \times 2$ & $2 \times 2 \times 144$ \\
\hline & 2 & $3 \times 3$ & $3 \times 3 \times 144$ \\
\hline & 3 & $4 \times 4$ & $4 \times 4 \times 144$ \\
\hline & 4 & $5 \times 5$ & $5 \times 5 \times 144$ \\
\hline & 5 & $6 \times 6$ & $6 \times 6 \times 144$ \\
\hline & 6 & $7 \times 7$ & $7 \times 7 \times 144$ \\
\hline
\end{tabular}

